\title{
Application of biochemical markers CA 19-9, CEA and C-reactive protein in diagnosis of malicious and benign pancreatic tumors
}

Jacek A. Śmigielski ${ }^{1}$, Łukasz Piskorz ${ }^{1}$, Marcin Wawrzycki ${ }^{1}$, Przemysław Dobielski², Małgorzata Pikala ${ }^{3}$, Sławomir Jabłoński ${ }^{1}$, Marian Brocki ${ }^{1}$

1Department of Thoracic Surgery, General Surgery and Oncology, Medical University of Lodz, Poland

2Department of Anesthesiology, Medical University of Lodz, Poland

${ }^{3}$ Epidemiology and Biostatistics Unit, Medical University of Lodz, Poland

Submitted: 22 June 2011

Accepted: 5 December 2011

Arch Med Sci 2013; 9, 4: 677-683

DOI: 10.5114/aoms.2013.36899

Copyright @ 2013 Termedia \& Banach
Corresponding author:

Jacek Śmigielski MD, PhD

Department of Thoracic

Surgery, General Surgery and Oncology

Medical University of Lodz

113 Żeromskiego St

90-549 Lodz, Poland

Phone: +48 426393521

E-mail: smiglo@mp.pl

\begin{abstract}
Introduction: We would save many lives and spare a lot of suffering if we could only detect and accurately determine the character and TMN staging of pancreatic tumors (PTs). With improved diagnosis, we could offer specific treatment that would result in better treatment outcome. The aim of study was to determine the significance of neoplastic markers CA 19-9 and CEA for prognosis in inflammatory and carcinomatous PTs.

Material and methods: We based our research upon a group of 170 patients. The patients were treated in our Oncologic Surgery Department from January 2007 to December 2010 for PTs. The patients were divided into four groups depending on the character of the tumor and underwent the following treatments: group $1-34$ patients with carcinoma of the ampulla of Vater, group 2 - 64 patients with PTs at different stages $(1,2,3)$ according to TMN classification, group $3-62$ patients with PTs at stage 4 on the TMN scale (unresectable tumors), group $4-28$ patients with inflammatory PTs.

Results: The results of Ca 19-9 in group 2 were 736.00 (25-75\% 220.40-4285.00) $\mathrm{ng} / \mathrm{ml}$ before surgery, $53.00(25-75 \% 12.60-84.00) \mathrm{ng} / \mathrm{ml}$ in the 7 days after surgery, $29.4(25-75 \% 7.90-113.00) \mathrm{ng} / \mathrm{ml}$ at day 30 , and 119.00 (25-75\% 96.30$621.00) \mathrm{ng} / \mathrm{ml} 3$ months after the operation. These results were significantly higher than the control group but were significantly lower than the results for group 3 (unresectable tumors). The highest average concentration and median for CA 19-9 and CEA were noted in patients with unresectable PTs (the $3^{\text {rd }}$ group). The average concentration for CEA was lowest in group 4, but much higher than the lab limits.

Conclusions: The sensitivity of the CA 19-9 marker may be as high as $88 \%$. Values of CA 19-9 above $852 \mathrm{U} / \mathrm{ml}$ may indicate TNM stage 4, consistent with an unresectable PT. In the cases where CA 19-9 is within normal limits but C-reactive protein is above normal limits (often thirty times the upper limit), in comparison to the control group and to patients with pancreatic neoplasms, strong consideration should be given towards the inflammatory characteristics of the pancreatic changes and conservative treatment should be applied.
\end{abstract}

Key words: CA 19-9, CEA, pancreatic tumors. 


\section{Introduction}

Difficulties in detecting and determining the character of pancreatic tumors (PTs) often result in poor treatment and outcome. Our goal is to detect PTs in a timely manner before they encroach upon biliary tracts or metastasize. In these scenarios we can only offer palliative surgery to relieve pain and overwhelming icterus [1].

It is hard to believe that despite the vast array of diagnostic tools we have at our disposal, a surgeon performing a pancreatic tumor procedure does not know exactly what to expect. Some of the perplexing questions they face are:

- Is the tumor benign or malignant?

- Is the neoplasm resectable or not?

- If the PT turns out to neoplastic, what is its tumor metastasis node (TMN) staging?

It goes without saying that different tumors require different surgical and anesthesiological (central line, TEA) approaches, but the final decision as to the extent of the surgery is made intraoperatively, often complicating the matter even further. Last, but not least, is the fact that we often add pain to misery if during explorative laparotomy we abandon the operation due to the unresectable nature of the tumor (TMN 4).

Data from the USA indicate that the incidence of pancreatic cancers is 8 to 12 per 100000 people per year. In total, there are about 32000 cases of this disease annually in the USA. Pancreatic cancer is the fourth leading cause of mortality from neoplastic disease in the USA, despite constituting only $3 \%$ of all cancers [2].

The epidemiology of pancreatic tumors is similar in Poland. There are roughly 3500 cases every year and they constitute $2.5 \%$ of all carcinomas. On average, upon resection the patient's life expectancy is around 24 months and it is shortened to 11 weeks in cases where palliative procedures alone are carried out [3-5].

Table I. Clinical use of tumor markers

\begin{tabular}{|lcc|}
\hline No. & Tumor type & Tumor marker \\
\hline 1 & Pancreatic cancer & Ca 19-9 \\
\hline 2 & Colorectal cancer & CEA, Ca 19-9 \\
\hline 3 & Breast cancer & Ca 15-3 \\
\hline 4 & Cervical cancer & SCC-Ag \\
\hline 5 & Prostate cancer & PSA, PAP \\
\hline 6 & Hepatocellular cancer & AFP \\
\hline 7 & Gastric cancer & Ca 19-9, CEA \\
\hline 8 & Lung cancer & NSE, SCC-Ag, Cyfra 21-1, CEA \\
\hline 9 & Testis tumor & Beta HCG, AFP, PLAP \\
\hline 10 & Ovarian tumor & Ca-125, $\beta$-HCG, AFP, CEA \\
\hline 11 & Thyroid cancer & Thyroglobulin, calcitonin \\
\hline
\end{tabular}

Signs and symptoms of PTs are few and far between and often only manifest when it is too late for intervention. The main symptoms are icterus (82\%), Gl discomfort (32\%), anorexia and weight loss (29\%), pruritus (21\%), nausea and vomiting or diarrhea often leading to cachexia and death. Often the very first sign of the illness is upper gastrointestinal bleeding. Even after diagnostic procedures such as computed tomography (CT), magnetic resonance imaging (MRI), and ultrasound, there is still a lack of certainty of definitive diagnosis with PTs. Every patient should be approached as an oncological case until proven otherwise [6-8].

The above-mentioned signs and symptoms of PTs are usually late and nonspecific and, therefore, the positive long-term treatment results for this illness are poor. Efforts are underway to find sensitive and specific lab biomarkers for PTs, allowing for prediction of the character of the tumor. When these biomarkers are found, we will be able to tell in advance whether the PT is resectable and the type of surgical procedure that will offer the best results. Unfortunately, we can only determine the character of the PT upon histological examination of the removed tumor mass, which is usually too late. Upon laparotomy we often find that the PT is unresectable and we resort to a palliative procedure restoring efflux of bile and sympathectomy for pain relief. The main difference between procedures now versus those in the eighties is the application of laparoscopic removal of PTs instead of open laparotomy [9].

It should also be noted that the two primary goals of palliative treatment are often accomplished by different methods, namely by endoscopic retrograde cholangiopancreatography (ERCP) (bile efflux) and video-assisted thoracoscopic sympathectomy (VATS) (sympathectomy) [10].

Although a number of lab biomarkers have already been tested as predictors of PTs, few are of any importance (Table I). Our main objective was to determine whether the concentration of the neoplastic markers carcinoma antigen 19-9 (CA 19-9) and carcinoembryonic antigen (CEA) can distinguish PTs versus inflammatory changes in the pancreas.

\section{Material and methods}

From January 2007 to December 2010 (4-year period), we enrolled 170 patients who were diagnosed with PTs which included benign, malignant, and inflammatory tumors. All of these patients underwent routine bedside examinations (physical, labs) and went through a series of abdominal cavity examinations as well as USG, ERCP, magnetic resonance cholangiopancreatography (MRCP) and/ or MRI.

Upon finishing treatment, we divided the patients into four groups based upon lab tests and 
histopathological results (done only upon removal of PTs):

- group 1 - 34 patients with carcinoma of the ampulla of Vater,

- group $2-64$ patients with PTs at different stages

$(1,2,3)$ according to TMN classification,

- group 3-62 patients with PTs at stage 4 on the TMN scale (unresectable tumors),

- group 4-28 patients with inflammatory PTs.

In group 3 the following treatments were performed: surgical bypass with anastomosis, including gastrojejunostomy or mixed gastrojejuno- and cholecysto-jejunostomy (triple bypass).

Blood samples $(2 \mathrm{ml})$ were taken through the antecubital vein on the day of the operation and then at 7, 30 and 90 days after the operation in all groups and the levels of CEA and CA 19-9 were determined. The blood was centrifuged and stored at $-60^{\circ} \mathrm{C}$. Levels of CEA, CA 19-9 and CRP were determined by standard electrochemiluminescence assay (ECLIA), method by means of Roche (CEA Kit No. 11731629322, CA19-9 Kit No. 11776193122, CRP Kit No. 11761428622). The study was performed using a Cobas 601 device. The patients were fasting at the time of sampling.

The fifth group was a control group composed of 20 patients who suffered from asymptomatic gallstones and were scheduled to have a cholecystectomy. The presence of any neoplastic disease in these patients was excluded. The blood samples were taken in this group in the same manner as described above.

Patients with active endocrine tumors of the gastroenteropancreatic neuroendocrine tumor (GEP-NET) type were also excluded from the research.

We were only able to follow up $57 \%$ of the initially enrolled patient population. Their medical records were scrutinized thoroughly. The remaining patients either did not report to the clinic or their family and friends refused to share any relevant information about them.

All patients signed informed consent and ethical committee approval no. RNN/592/11/KB was obtained.

\section{Statistical analysis}

Statistical analysis was carried out with the help of Statistica 9.5. ANOVA and post hoc tests were used to check that results in all five groups were correct and had statistical significance. The statistical differences between values of CA 19-9 and CEA were checked periodically (see above) and compared with Freidman's ANOVA test. The results were also scrutinized based on ROC (receiver operating characteristic) curves showing linear interdependencies between sensitivity and specificity for each of the markers (CEA and CA 19-9). A median was applied to compare results given scattering of the data and count of the groups.

\section{Results}

After analysis, the level of the highest median of CA 19-9 recorded in group 3 was 4578.00 (25-75\% 1456.00-9600.00) ng/ml before surgery, 4,761.00 (25-75\% 1680.00-10,560) ng/ml in the 7 days after surgery, 5730.00 (25-75\% 2300.00-11805.00) ng/ml at day 30, and 9600.00 (25-75\% 4765.00-14200.00) $\mathrm{ng} / \mathrm{ml} 3$ months after surgery. The results from group 3 were significantly different than those from the control group and the other groups (Tables II and III).

From a clinical point of view, the greatest practical value is in the results of CA 19-9 in group 2. The levels from group 2 were 736.00 (25-75\% 220.40-4285.00) ng/ml before surgery, 53.00 (25$75 \% 12.60-84.00) \mathrm{ng} / \mathrm{ml}$ in the 7 days after surgery, $29.4(25-75 \% 7.90-113.00) \mathrm{ng} / \mathrm{ml}$ at day 30 , and 119.00 (25-75\% 96.30-621.00) $\mathrm{ng} / \mathrm{ml} 3$ months after the operation. These results were significantly higher than those in control group 4 and significantly lower than in group 3 . There were no statistically significant differences in comparison with group 1. The level of the highest median of CRP was recorded in group 4 (Table IV). The summary of CEA median results is shown in Table $\mathrm{V}$. The results from group 3 were significantly different than those from the control group and the other groups. The sensitivity of CEA was 0.462 and specificity $0.7(\mathrm{AUC}=0.727)$; however, sensi-

Table II. Median concentration of CA 19-9 in the studied groups of patients

\begin{tabular}{|lccccc|}
\hline Before surgery & \multicolumn{3}{c|}{ CA 19-9 } \\
\cline { 2 - 5 } & Median & $25-75 \%$ & $p^{*}$ & $p^{\star *}$ & $p^{\star \star *}$ \\
\hline Carcinoma of the Ampulla of Vater & 55.150 & $16.50-110.30$ & & $p^{\star \star \star *}$ \\
\hline PTs at different stages $(1,2,3)$ & 736.000 & $220.40-4285.00$ & & 0.0022 \\
\hline PTs at stage 4 & 4578.000 & $1456.00-9600.00$ & 0.0087 & $<0.001$ \\
\hline Inflammatory PTs & 24.700 & $8.80-61.80$ & & 0.027 \\
\hline Control group & 13.30 & $6.30-16.00$ & & & $<01$ \\
\hline
\end{tabular}

${ }^{*}$ Comparison with carcinoma of the ampulla of Vater, ${ }^{* *}$ comparison with PTs at different stages $(1,2,3),{ }^{* * *}$ comparison with inflammatory PTs, ${ }^{* * * *}$ comparison with control group 
Table III. Median concentrations of CA 19-9 in 90 days of study

\begin{tabular}{|lcccccc|}
\hline \multirow{2}{*}{90 days } & \multicolumn{5}{c|}{ CA 19-9 } \\
\cline { 2 - 6 } & Median & $25-75 \%$ & $p^{*}$ & $p^{\star \star}$ & $p^{\star \star \star}$ & $p^{\star \star \star \star *}$ \\
\hline Carcinoma of the ampulla of Vater & 29.10 & $12.60-63.50$ & & & & \\
\hline PTs at different stages $(1,2,3)$ & 119.00 & $96.30-621.00$ & & & \\
\hline PTs at stage 4 & 9600.00 & $4765.00-14200.00$ & $<0.001$ & 0.0483 & $<0.001$ & $<0.001$ \\
\hline Inflammatory PTs & 27.15 & $7.50-63.54$ & & & & \\
\hline Control group & 13.30 & $6.30-16.00$ & & 0.0311 & & \\
\hline
\end{tabular}

${ }^{*}$ Comparison with carcinoma of the ampulla of Vater, ${ }^{* *}$ comparison with PTs at different stages $(1,2,3),{ }^{* * *}$ comparison with inflammatory PTs, ${ }^{* * * *}$ comparison with control group

Table IV. Median CRP levels before the surgery

\begin{tabular}{|c|c|c|c|c|c|c|}
\hline \multirow[t]{2}{*}{ Before surgery } & \multicolumn{6}{|c|}{ CRP } \\
\hline & Median & $25-75 \%$ & $p^{*}$ & $p^{\star *}$ & $p^{\star \star \star}$ & $p^{\star \star \star *}$ \\
\hline Carcinoma of the ampulla of Vater & 70.00 & $23.80-92.00$ & & & & \\
\hline PTs at different stages $(1,2,3)$ & 38.70 & $11.00-74.00$ & & & & \\
\hline PTs at stage 4 & 48.90 & $26.70-85.80$ & & & 0.043 & 0.0244 \\
\hline Inflammatory PTs & 166.40 & $109.00-267.00$ & & 0.0248 & & \\
\hline Control group & 4.95 & $3.5-6.1$ & & & $<0.001$ & \\
\hline
\end{tabular}

${ }^{*}$ Comparison with carcinoma of the ampulla of Vater, ${ }^{* *}$ comparison with PTs at different stages $(1,2,3),{ }^{* * *}$ comparison with inflammatory PTs, ${ }^{* * * *}$ comparison with control group

Table V. Median CEA levels before the surgery

\begin{tabular}{|c|c|c|c|c|c|c|}
\hline \multirow[t]{2}{*}{ Before surgery } & \multicolumn{6}{|c|}{ CEA } \\
\hline & Median & $25-75 \%$ & $p^{*}$ & $p^{\star \star}$ & $p^{\star \star \star}$ & $p^{\star \star \star *}$ \\
\hline Carcinoma of the Ampulla of Vater & 2.55 & $1.78-3.50$ & & & & \\
\hline PTs at different stages $(1,2,3)$ & 4.10 & $2.15-6.04$ & & & & \\
\hline PTs at stage 4 & 13.93 & $17.76-26.80$ & $<0.001$ & & $<0.001$ & $<0.001$ \\
\hline Inflammatory PTs & 3.25 & $1.78-4.25$ & & & & \\
\hline Control group & 2.55 & $1.70-3.60$ & & & & \\
\hline
\end{tabular}

${ }^{*}$ Comparison with carcinoma of the ampulla of Vater, ${ }^{* *}$ comparison with PTs at different stages $(1,2,3),{ }^{* * *}$ comparison with inflammatory PTs, ${ }^{* * * *}$ comparison with control group

tivity of CA $19-9$ was 0.889 and specificity 0.9 $(A \cup C=0.9)($ Table $V)$.

\section{Discussion}

Today worldwide, up to $30 \%$ of operations performed on patients with pancreatic tumors are "unnecessary" [5]. Some represent operations carried out in patients with benign tumors and others are laparotomies in patients with stage 4 malignancy. At present, we have no means by which to distinguish benign from malignant tumors pre-surgically with $100 \%$ certainty. Knowing even a single ideal biomarker that can answer the question of whether the patient has pancreatic cancer before surgery would allow for the exclusion of more than $30 \%$ of these procedures, which ultimately should not be performed.

Suspicion of a specific cancer would denote a particular level of a corresponding biomarker in order to confirm or exclude the disease (sometimes more than one, to differentiate between tumors), while determination of a panel of markers in every case of suspected malignancy is not advisable [10, 11].

When diagnosing pancreatic cancer, patients must fulfill criteria or risk factors for the disease. Today, a confirmed and well-known risk factor for pancreatic cancer is nicotine and occupational exposure to certain chemicals (benzidine and $\beta$-naphthylamine) [12-14]. Currently, the best known and examined pancreatic cancer marker is CA 19-9. The characteristics of an ideal marker are high sensitivity, high specificity, predictive value and specificity of the organ [15].

Specificity is the proportion of patients who are normal or have benign disease, in whom the values obtained in the test are negative (i.e. below the threshold). A lower percentage of false positives results in a higher specificity for the marker. Speci- 
ficity tells us about the ability of a test to identify healthy individuals [16].

In contrast, sensitivity is the proportion of patients with cancer, in whom the values obtained in the test are positive. The lower the percentage of false-negative results, the higher the sensitivity of the marker. Sensitivity is the ability of the test to detect disease. CA 19-9 has been shown to have a specificity of approximately $85 \%$ and a sensitivity of $90 \%[15,17]$. In our study, the sensitivity is $88 \%$. Therefore, the search for the perfect marker persists. At present, we know the standards, the specificity and sensitivity of the marker, but we do not know if exceeding the normal range, e.g. 2 or 4-fold, may indicate an unresectable tumor.

The surgeon could allow the possibility to gauge preoperatively whether surgery should be performed and possibly allow distinction between benign tumors of the pancreas and those that are contraindicated for surgical removal. The tumor stage gives the possibility of radical surgery and a potentially good prognosis or disqualifies the patient from surgery and does not expose the patient to operative trauma as well as the possibility of complications.

The prognostic value of CA 19-9 as a marker of survival in pancreatic cancer is higher postoperatively rather than preoperatively [18]. It was also found that level of CA 19-9 is an independent prognostic factor [19]. Reni et al. [20] studied a group of patients undergoing neoadjuvant chemotherapy in advanced pancreatic cancer, depending on the level of CA 19-9. Average concentration of CA 19-9 serum levels above $1167 \mathrm{U} / \mathrm{ml}$ allows for survival of only 8 months.

CA 19-9 is also used in the differentiation between benign and malignant intraductal papillary mucinous neoplasms. High sensitivity, close to $86 \%$, makes it possible to predict malignant change



Figure 1. ROC curve for CA 19-9 and CEA before operations in the control group and in patients with operable pancreatic tumor
$[21,22]$. But $86 \%$ does not give certainty. The question is how to proceed? Despite a lack of diagnosis of malignant changes, should we expose the patient to significant treatment, or watch and wait, allowing the possible progression of cancer?

In all doubtful or controversial cases, those with lack of a diagnosis of malignant change, or in the presence of cysts or cystic glandular changes, UK authors recommend aggressive surgical management [23] with all its ensuing consequences.

After analyzing our results, we found a significantly higher median level of CA 19-9 before surgery in patients with inoperable pancreatic cancer compared to the other groups (average concentration of $6230 \mathrm{U} / \mathrm{ml}$ and a median of $4578 \mathrm{U} / \mathrm{ml}$ ). As the number of days increased ( $7^{\text {th }}$ day - average median of $4761 \mathrm{U} / \mathrm{ml}$ ) up to 90 days (average median $9630 \mathrm{U} / \mathrm{ml}$ ) the average median increased. Such high values at any point are considered strong evidence of the progress of the disease.

However, there were significantly higher median levels of CA 19-9 in patients with operable pancreatic cancer in relation to the control group before treatment (mean concentration of $852 \mathrm{U} / \mathrm{ml}$ and a median of $736 \mathrm{U} / \mathrm{ml}$ ) and these levels decreased in the subsequent postsurgical period with a decrease of 7 to 30 times with no statistical difference. At 3 months after surgical resection, the median CA 19-9 levels were again significantly different compared with the control group. There may be a temporary regression of the resected tumor which could possibly be caused by local recurrence and/or changes in the site of metastasis. The sensitivity and specificity of CA 19-9 in the control group compared to the group of patients with resectable pancreatic cancer in the initial phase of the study was high, reaching 90-92\% with a cut-off point of $32 \mathrm{U} / \mathrm{ml}$, with an AUC of 0.9 that respectively decreased over time after surgery between $32-53 \%$,

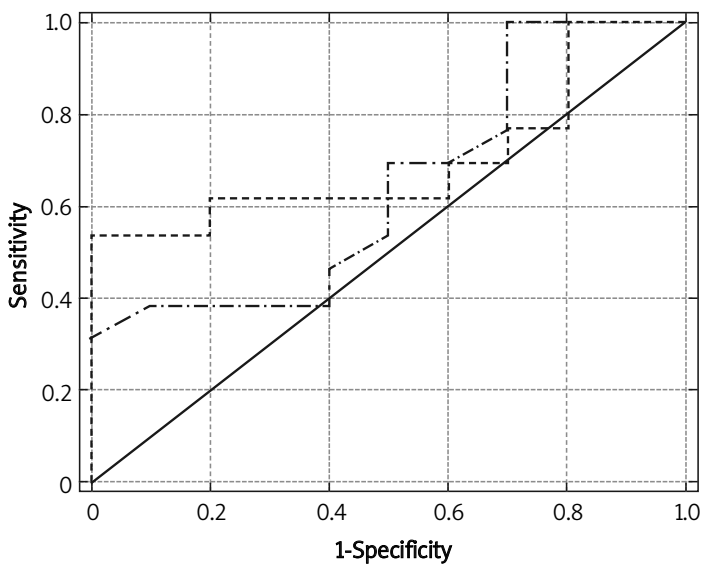

--- CEA_30 --CA 19-9_30 —-Baseline

Figure 2. ROC curve for CA $19-9$ and CEA after 30 days of testing in the control group and in patients with operable pancreatic tumor 
and an AUC of 0.642 for the same cut-off point at day 30 (Figure 2). This may be evidence of the effectiveness of surgical treatment. Histopathological findings were analyzed in 4 cases (9\%) which had $\mathrm{R} 1$ resection and the remaining $\mathrm{R} 0$. We will discuss this topic in a future study.

Interesting observations can be drawn by analyzing the group of patients with nonmalignant pancreatic tumors. Determination of preoperative CA 19-9 and CEA with no statistical differences compared to the control group and significantly higher median concentrations of CRP (normal value exceeded more than 30 -fold) may indicate the presence of an inflammatory tumor of the pancreas (Table IV).

A Polish research group [24] looked for an ideal marker using matrix metalloproteinases (MMP), proteolytic enzymes involved in the processes of angiogenesis and collagen fiber structure of the extracellular matrix along with their inhibitors TIMP. It was found that MMP may be an independent predictor of survival in patients with pancreatic cancer.

There is also ongoing research of the carcinoembryonic antigen and its role in pancreatic cancer. Its specificity is low. Pitman et al. [25] concluded that the presence of atypical cells in the fluid of a pancreatic cyst has more prognostic significance than the concentration of CEA in the fluid with values greater than $2500 \mathrm{ng} / \mathrm{ml}$. Our study confirms a low clinical utility of this marker in the diagnosis and differential diagnosis of pancreatic neoplasms. CEA values were significantly higher throughout the study period only in patients with unresectable cancer, which may indicate the existence of metastases, e.g. to the liver, during the simultaneous diagnosis of disease in the pancreas.

In conclusion, The highest average and median concentration of CA 19-9 and CEA are present in patients with unresectable pancreatic cancer. Average median CEA concentration was lowest in patients with inflammatory tumors, but significantly higher than that of normal patients. The sensitivity of the marker CA 19-9 in malignant tumors of the pancreas reaches $88 \%$. The average concentration of the marker CA 19-9 above $852 \mathrm{U} / \mathrm{ml}$ may be a stage 4 cancer and unresectable pancreatic tumor. In cases where CA 19-9 is within normal limits but CRP is above normal limits (often thirty times over the upper limit) in comparison to the control group and to patients with pancreatic neoplasms, strong consideration should be given to the inflammatory characteristics of the pancreatic changes and conservative treatment should be applied.

\section{References}

1. Roehrig S, Wein A, Albrecht $\mathrm{H}$, et al. Palliative first-line treatment with weekly high-dose 5 -fluorouracil as $24 \mathrm{~h}$ - infusion and gemcitabine in metastatic pancreatic cancer (UICC IV). Med Sci Monit 2010; 16: CR124-131.

2. Popiela T, Drews M, Jeziorski A, et al. Tumors of the stomach, ampulla of Vater, small intestine, pancreas, liver, bile ducts and gallbladder. Via Medica, Gdansk 2009.

3. Cichocki A, Jóźwiak M, Samsel R, Piotrowski P, Nawrocki G, Roszkowska K. Characteristics of tumors of the pancreas and duodeno-pancreatic field in own material in a prospective 2-year follow-up. Pol. Przeg Chir 2008; 80: 415-27.

4. Lampe P, Kuśnierz K. Comments on selected issues of pancreatic surgery. Pol Przeg Chir 2008; 80: 527-32.

5. Jesipowicz J, Piłat J, Jesipowicz M, Matras P, Rudzki S. Late detection, progress and results of treatment of pancreatic cancer. Wspolczesna Onkol 2000; 4: 76-9.

6. Ji J, Forsti A, Sundquist J, Lenner P, Hemminki K. Survival in familial pancreatic cancer. Pancreatology 2008; 8: 252-6.

7. Hać S, Dobrowolski S, Śledziński Z. Pancreatic-gastric anastomosis after the duodenum and pancreatic head resection. Pol Przeg Chir 2008; 80: 687-93.

8. Finlayson $\mathrm{E}, \mathrm{Clark} \mathrm{OH}$. Surgery treatment of pancreatic neuroendocrine tumor. Surg Clin N Am 2004; 84: 775-85.

9. House MG, Choti MA. Palliative treatment of pancreatic and bile duct cancer. Surg Clin N Am 2005; 85: 359-71.

10. Śmigielski J, Hołyński J, Kococik M, et al. Paliative procedures in cholangiocarcinomas - experience of 5 centers. Pol Merkuriusz Lek 2009; 155: 416-9.

11. Wan C, Shen Y, Yang T, Wang T, Chen L, Wen F. Diagnostic value of microRNA for pancreatic cancer: a meta-analysis. Arch Med Sci 2012; 8: 749-55.

12. lodice S, Gandini S, Maisonneuve P, Lowenfels AB. Tobacco and the risk of pancreatic cancer: a review and metaanalysis. Langenbecks Arch Surg 2008; 393: 535-45.

13. Lindkvist B, Appelros S, Manjer J, Berglund G, Borgstrom A. A prospective cohort study of smoking in acute pancreatitis. Pancreatology 2008; 8: 63-70.

14. Hassan MM, Bondy ML, Wolff RA, et al. Risk factors for pancreatic cancer: case-control study. Am J Gastroenterol 2007; 102: 2696-707.

15. Rudnicki J, Agrawal AK, Grzebieniak Z, et al. Prognostic value of CA 19-9 level in resectable pancreatic adenocarcinoma. Folia Histochem Cytobiol 2010; 48: 249-61.

16. Maisey NR, Norman AR, Hill A, Massey A, Oates J, Cunningham D. CA19-9 as a prognostic factor in inoperable pancreatic cancer: the implication for clinical trials. Br J Cancer 2005; 93: 740-3.

17. Joergensen MT, Brunner N, Schaffalitzky De Muckadell OB. Comparison of circulating MMP-9, TIMP-1 and CA19-9 in the detection of pancreatic cancer. Anticancer Res 2010; 2: 587-92.

18. Kondo N, Murakami Y, Uemura K, et al. Prognostic impact of perioperative serum CA 19-9 levels in patients with resectable pancreatic cancer. Ann Surg Oncol 2010; 17: 2321-9.

19. Jaeschke R, Cook D, Guyatt G. Methods for determining the suitability of the test. Med Prakt 1999; 1: 63.

20. Reni M, Cereda S, Balzano G, et al. Carbohydrate antigen 19-9 change during chemotherapy for advanced pancreatic adenocarcinoma. Cancer 2009; 115: 2630-9.

21. Fritz S, Hackert T, Hinz U, Hartwig W, Büchler MW, Werner J. Role of serum carbohydrate antigen 19-9 and carcinoembryonic antigen in distinguishing between benign and invasive intraductal papillary mucinous neoplasm of the pancreas. Br J Surg 2011; 98: 104-10.

22. Mimura T, Masuda A, Matsumoto I, et al. Predictors of malignant intraductal papillary mucinous neoplasm of the pancreas. J Clin Gastroenterol 2010; 44: 224-9. 
23. Jones NB, Hatzaras I, George N, et al. Clinical factors predictive of malignant and premalignant cystic neoplasms of the pancreas: a single institution experience. HPB (Oxford) 2009; 11: 664-70.

24. Mroczko B, Lukaszewicz-Zajac M, Wereszczynska-Siemiatkowska $U$, et al. Clinical significance of the measurements of serum matrix metalloproteinase- 9 and its inhibitor (tissue inhibitor of metalloproteinase-1) in patients with pancreatic cancer: metalloproteinase- 9 as an independent prognostic factor. Pancreas 2009; 38: 613-8.

25. Pitman MB, Michaels PJ, Deshpande V, Brugge WR, Bounds BC. Cytological and cyst fluid analysis of small ( $<$ or $=3 \mathrm{~cm}$ ) branch duct intraductal papillary mucinous neoplasms adds value to patient management decisions. Pancreatology 2008; 8: 277-84. 